\title{
COMO AS ESTRATÉGIAS DE COMBATE À CORRUPÇÃO ADOTADAS PELOS TRIBUNAIS DE CONTAS ESTADUAIS DO BRASIL SÃO INFLUENCIADAS PELA RENDA PER CAPITA?
}

\section{How the strategies to fight corruption adopted by Brazilian's State Audit Courts are influenced by per capita income?}

\section{Robson Fernandes Soares}

Doutorando em Administração. Universidade Positivo. Curitiba, PR. Brasil.e-mail:robsoares88@gmail.com

\section{Rodolfo Coelho Prates}

Doutor em Economia Aplicada pela Universidade de São Paulo. Professor visitante do Middlebury College. Curitiba, PR. Brasil. e-mail:rodprates@hotmail.com

\section{Márcio José Assumpção}

Mestre em Administração pela Universidade de Extremadura. Professor na Universidade Positivo. Curitiba, PR. Brasil. e-mail:mja@tce.pr.gov.br

\section{RESUMO}

Embora as Instituições Supremas de Auditoria sejam reconhecidas como um dos pilares de combate à corrupção, pouco se sabe a respeito de como elas concebem seu modo de atuação e como consideram as variáveis ambientais em seu planejamento. Dessa forma, o objetivo do presente estudo consiste em compreender como a formulação estratégica dessas entidades leva em consideração a renda per capita e a desigualdade de renda (principais variáveis ambientais relacionadas à corrupção). Para tanto, foram analisados os planejamentos estratégicos de 24 Tribunais de Contas Estaduais que se encontravam vigentes em 2015. Através da análise de conteúdo, foram identificadas sete dimensões estratégicas que foram relacionadas aos níveis de renda e de desigualdade de renda através da Análise de Homogeneidade. Em seguida, foi aplicado o teste exato de Fisher para confirmar as relações levantadas. Como principal resultado, verificou-se que estratégias de capacitação da gestão pública estão relacionadas com contextos de renda elevada.

Palavras-chave: Planejamento estratégico. Estratégias. Corrupção. Renda per capita. Tribunal de Contas.

\section{ABSTRACT}

Although the Supreme Audit Institutions (SAIs) are recognized as one of the pillars in the fight against corruption, there is no knowledge enough about how they conceive their way of acting and how they take in account environmental factors in their planning to combat corruption. Thus, the objective of this study is to understand how this strategy formulation consider per capita income and income inequality (main environmental variables related to corruption). Therefore, we analyzed the strategic plans of 24 State Audit Courts for the year 2015. Through Content Analysis (CA) were identified seven strategic dimensions that were related to income levels and income inequality by Homogeneity Analysis (Homals). Then we applied the Fisher's exact test (statistic test) to confirm relations that was raised. The main result was that training strategies of public management are related to high-income contexts.

Keywords: Strategic plan. Strategies. Corruption. Per capita income. Audit institutions. 


\section{INTRODUÇÃO}

A corrupção tem sido apontada pela literatura como um fator que freia o crescimento econômico dos países, isso porque elevados índices de corrupção são vistos como fatores de instabilidade política que repelem os investimentos, incluindo o investimento estrangeiro direto (MAURO, 1995). Também tem sido destacado o aspecto negativo da corrupção na produtividade, pois ela faz com que sejam escolhidos os projetos que ofereçam a maior quantidade de propina ao invés daqueles com o melhor alcance (LAMBSDORFF, 2003). Esses agravamentos têm feito com que os países busquem mecanismos de combate à corrupção para melhorar sua inserção internacional, seja por vontade própria ou por pressão de órgãos, como o Fundo Monetário Internacional (FMI) e o Banco Mundial, por exemplo.

A literatura também apresenta uma série de alternativas anticorrupção, algumas defendendo ações intervencionistas, outras gerencialistas e ainda algumas reforçando o desenvolvimento da integridade organizacional (MCCUSKER, 2006). A efetividade dessas estratégias, contudo, ainda não é bem clara. Há um certo consenso de que o contexto deve ser levado em consideração no design estratégico. Nesse sentido, há um número de estratégias que apresentam efetividade apenas para países de baixa renda (HUBERTS, 1998).

Para esses países, há evidências de que a taxa de corrupção é mais elevada do que a dos países com maior renda per capita, principalmente em função da diferença no capital humano (SVENSSON, 2005). O que indica que nos países de baixa renda a corrupção seja vista como um fenômeno muito mais estrutural e ligado a um conceito moral de corrupção, do que a um problema de agência.

Contudo, em que pese as recomendações da literatura a respeito dos mecanismos de combate à corrupção, as nações diferem muito quanto aos meios que adotam (STAPENHURST; KPUNDEH, 1999). Em boa parte são influenciadas por pressões internacionais, mas também há um processo de resistência em que se acredita que as mudanças institucionais geradas por pressões internacionais são guiadas por interesses de credores preocupados na solvência de curto prazo e não com um compromisso de longo prazo com o país (MARTONE, 2007).

Fato é que ainda se conhece pouco a respeito de como essas estratégias são formuladas, principalmente em países em desenvolvimento. Nesse sentido, o presente estudo tem a seguinte pergunta de pesquisa: como as estratégias de combate à corrupção adotadas pelos Tribunais de Contas Estaduais Brasileiros são influenciadas pela renda per capita e pelo coeficiente de Gini de cada Estado?

No Brasil existem 27 Tribunais de Contas Estaduais, sendo um em cada unidade da Federação. Eles têm como missão constitucional exercer o controle externo no âmbito da administração pública de cada jurisdição. Esses órgãos são peças fundamentais para enfrentar a corrupção e conferir legitimidade institucional ao país, pois têm como atribuição garantir o cumprimento da vontade do Legislativo. Também são responsáveis por promover programas de prevenção e combate à corrupção. Portanto, as estratégias que adotam podem ser entendidas como um reflexo da vontade do Legislativo a que representam.

Eles estão localizados em contextos econômicos muito diversificados, estando alguns em regiões com alta renda per capita e outras com baixa renda per capita. Apesar disso, possuem certa homogeneidade histórica, cultural, legal e religiosa, que faz com que se possa estudar a relação entre renda per capita e estratégia de uma forma menos problemática que em comparações entre países.

Na sequência é apresentado um referencial teórico no qual se discute o papel dessas instituições de auditoria governamental, bem como o processo de formulação estratégica das mesmas. Em seguida são apresentadas considerações sobre como a renda per capita pode ser considerada como variável ambiental.

\section{REFERENCIAL TEÓRICO}

Nessa seção, em um primeiro momento, se discutem as mudanças na forma de atuação estratégica das Instituições Supremas de Auditoria, principalmente no que diz respeito ao combate à corrupção. Em seguida são apresentadas considerações sobre como a variável renda está relacionada com a temática corrupção. Por fim, se discute como o contexto 
de pobreza pode estar relacionado com a formulação estratégica das Intuições Supremas de Auditoria.

\subsection{A Formulação Estratégica das Instituições Supremas de Auditoria (ISA)}

A literatura tem mostrado que a formulação estratégica das Instituições Supremas de Auditoria (ISA) apresenta grande variação ao redor do mundo. Em alguns países há uma orientação para uma atuação gerencialista, através de auditorias de desempenho. Já em outros países prevalece a realização de auditorias de conformidade (BARZELAY, 1997). É reconhecido que parte dessa orientação provém da organização estrutural da própria ISA. Nesse sentido, em países que prevalece um sistema de Corte de Contas, como no Brasil, há o predomínio de auditorias de conformidade, ao passo que em países que adotam um modelo de controladorias, como os Estados Unidos, há uma busca por auditorias de desempenho.

No que diz respeito ao combate à corrupção, o modelo de Corte de Contas tem sido visto como menos efetivo do que os demais modelos de entidades supremas de auditoria (BLUME; VOIGT, 2011). Essa baixa efetividade no combate à corrupção tem sido atribuída à baixa utilização dos relatórios por parte do Legislativo, à pouca divulgação dos casos de corrupção e à baixa efetividade das punições concedidas.

Esse contexto tem levado as Cortes de Contas a buscarem uma nova forma de atuação, combinando seu papel judicante a um papel orientativo. Dessa forma, elas podem agir diretamente buscando identificar desvios e reportando-os em seus relatórios, mas também é possível uma atuação indireta, em que essas instituições atuem fornecendo orientações para que todos os integrantes dos sistema de controle interno atuem melhor (KAYRAK, 2008). Nessa atuação indireta são possíveis ações que: a) promovam a transparência, accountability e a boa governança no setor público; b) aumentem a consciência do Executivo quanto às fragilidades de seus sistemas de controle; c) facilitem o acesso da mídia aos casos de corrupção.

Essas orientações para uma atuação mais ampla das ISA têm sido encampadas pela International Organisation of Supreme Audit Institutions (INTOSAI), que reconhece a influência dos valores sociais, tradi- ções e princípios de uma sociedade na ocorrência de corrupção, e que, embora as instituições supremas de auditoria possam fazer pouco para mudar a sociedade, elas devem considerar essas peculiaridades na hora de planejar sua forma de atuação (KELLNER, 2000). Dessa forma, violência, grau de pobreza, injustiça, entre outros fatores, devem ser considerados na elaboração do plano de auditoria.

Esse papel gerencialista e de atuação indireta, embora ainda residual e fortemente influenciado por uma tradição legalista, tem ganhado espaço nos países de Cortes de Contas, principalmente frente às críticas relacionadas à baixa efetividade dos relatórios e pela orientação voltada para indicadores de desempenho (JÚNIOR; DE AQUINO, 2011). Essas instituições estão mudando suas formas de atuação, buscando atender uma ampla gama de usuários e ampliando seu rol de atividades. Além da típica função judicante, é comum encontrar Instituições de Auditoria exercendo papéis de consultoria aos jurisdicionados e de pesquisa no setor público, assim como agente de divulgação de dados do setor público (POLLITT, 2003). Nesse sentido, são emergentes novas estratégias de atuação (BRINGSELIUS, 2014).

Nessa nova perspectiva, o enfoque passa de uma atuação repressiva para uma de caráter de orientação, com enfoque na mudança de comportamento. Ao lado das típicas irregularidades, os relatórios passam a trazer recomendações de melhoria dos sistemas de controle. Isso tem gerado um impacto positivo no comportamento dos auditados, mudando sua forma de atuação (JOHNSEN et al., 2001; REICHBORN-KJENNERUD; JOHNSEN, 2015).

Essa nova forma de atuação também parece atender de forma mais adequada ao Legislativo, que utilizam os relatórios muito mais com o propósito de promover a accountability, comunicação e meio de promoção da eficiência do que forma de punição (MCDAVID; HUSE, 2011).

\subsection{Corrupção, Renda e Desigualdade}

Os trabalhos empíricos têm encontrado uma correlação entre níveis de corrupção e nível de renda dos países. Parte da literatura tem argumentado que os elevados índices de corrupção são a causa do baixo nível de renda, uma vez que nos locais com 
maior corrupção há um desincentivo para atividade econômica (GUPTA et al., 2002). Nesse sentido, são encontradas evidências de que a implementação de políticas anticorrupção tem como efeito a diminuição das desigualdades e o aumento da renda per capita (WU; ZHU, 2011).

Por outro lado, há uma vertente que tem associado os níveis de pobreza como causa da corrupção. Isso porque os ambientes corruptos favoreceriam as elites, que têm melhores meios de sonegar tributos e maior facilidade de chegar à posições hierarquicamente superiores na administração pública, podendo, portanto, desviar recursos (JONG-SUNG; KHAGRAM, 2005). Por fim, também há quem argumente pela existência de um efeito bidirecional, no sentido de que a corrupção gera desigualdade e a desigualdade cria um ambiente propício à corrupção (NEGIN et al., 2010).

Também é reconhecido que o nível de renda está positivamente associado à corrupção (MOCAN, 2008). Isso ocorre porque, em geral, as pessoas com maior renda se encontram em cargos que movimentam maior quantidade de recursos e são responsáveis por decisões-chave, sendo, portanto, mais sujeitas à ofertas de suborno.

\subsection{As Estratégias e a Concepção de Corrupção}

Para entender as estratégias de combate à corrupção é necessário antes compreender a natureza do fenômeno corrupção. Essa discussão passa por uma concepção mais ampla sobre a natureza do próprio ser humano, que pode ser compreendido em uma perspectiva hobbeseniana, como um ser naturalmente egoísta, guiado apenas por interesses individuais, ou dentro de uma perspectiva moralista, em que se acredita que o ser humano possui um senso de coletividade, tendo um comportamento associado à estrutura social que pertence (BUKOVANSKY, 2006).

Essas duas noções de ser humano dão suporte a duas teorias sobre corrupção. A primeira delas (teoria utilitarista) entende que o ser humano é um agente econômico racional, e, dessa forma, maximiza os be- nefícios econômicos, além disso, ele é desprovido de considerações éticas. A segunda (teoria institucional) entende que, embora parte do comportamento seja guiado por interesses individuais, há uma parte do comportamento que não é explicado apenas dentro de uma lógica de racionalidade, havendo um componente moral que interfere na decisão do agente econômico (HODGSON; JIANG, 2007).

Nesse sentido, há algumas evidências de fatores não econômicos que também influenciam os níveis de corrupção. Alguns trabalhos mostram, por exemplo, que a orientação religiosa é um fator explicativo do nível de corrupção (PALDAM, 2001). Outros demonstram que quanto maior o nível de confiança entre os cidadãos, menores são os índices de corrupção e mais produtiva é a sociedade (PORTA et al., 1996).

Dentro desse contexto, pode-se pensar em estratégias ligadas ao combate de um comportamento oportunista e em estratégias associadas a um desenvolvimento de uma moral coletiva. As primeiras estão voltadas ao combate de um comportamento utilitarista, que é inerente a um pensamento racional do agente. Já as segundas estão relacionadas ao desenvolvimento de valores coletivos de cooperação.

Parece razoável supor que localidades com maior renda estão mais próximas dessa moralidade coletiva e que em localidades de baixa renda prevalece uma falsa moral, ou uma racionalização da moral.

Nesse sentido, em localidades de alta renda há maior acesso à educação, ao mercado de trabalho e menor desigualdade econômica, havendo, portanto, um menor incentivo para quebra do pacto social de moralidade. Já em uma localidade de baixa renda, o indivíduo se sente desamparado estruturalmente, vendo a corrupção como uma alternativa para escapar da pobreza. Assim, a quebra de um pacto social se justificaria em função de uma espécie de justiça social mais ampla, ainda que realizada por meio de práticas corruptas.

Dentro dessa linha, em sociedades mais ricas podem prevalecer estratégias predominantemente combativas, ao passo que nas sociedades mais pobres, além dessas, também se encontram presentes estratégias moralistas (orientativas). 


\section{PROCEDIMENTOS METODOLÓGICOS}

Nesta seção são discutidos o processo de coleta de dados, a construção das variáveis de constructo e operacionais, as premissas adotadas no processo de codificação e categorização, a hipótese de pesquisa e os métodos de análise.

\subsection{Coleta dos Dados}

Para realização da pesquisa foi utilizado o método de levantamento de dados documentais, utilizando-se de dados secundários, que foram coletados diretamente dos endereços eletrônicos das organizações em estudo.

Foram eleitos como corpus de análise os planejamentos estratégicos dos 27 Tribunais de Contas Estaduais. A escolha dos documentos de análise teve como base os critérios de exaustividade e homogeneidade propostos por Bardin (2009). Isso porque esses documentos são semelhantes (homogêneos) e é possível realizar uma cobertura completa do conteúdo dos mesmos (exaustiva).

Além disso, os planejamentos estratégicos constituem o meio formal pelo qual esses órgãos expressam suas intenções de ação, obedecendo a regra da pertinência. Já os demais documentos não representam um compromisso instucionalizado do órgão, podendo mais facilmente estar associado a um jogo de palavras em busca de legitimidade. Nos planejamentos estratégicos esse risco também é presente, contudo em grau menor, uma vez que há um desdobramento dos objetivos em metas das quais será exigida uma prestação de contas pela sociedade.

Optou-se por um corte transversal, abrangendo apenas os planejamentos estratégicos vigentes em 2015, uma vez que se tem por objetivo comparar a formulação estratégica em diferentes contextos regionais.

Procedeu-se a consulta dos planejamentos estratégicos constantes nos sites dos 27 Tribunais de Contas Estaduais, encontrando-se disponível em meio online 24 deles. Não foi possível localizar os planejamentos dos Tribunais de Contas dos Estados do Alagoas, Amapá e Mato Grosso do Sul. Para esses foi encaminhado um e-mail solicitando tal informação, mas não foi obtida resposta.

\subsection{Abordagem da Análise de Conteúdo (AC)}

Para poder comparar as diferentes estratégias de combate à corrupção adotadas pelos Tribunais de Contas Estaduais foi necessário recorrer a um método que desse suporte a um processo de categorização e sistematização de textos. Isso fez com que a Análise de Conteúdo tradicional fosse uma opção lógica, uma vez que essa técnica pode ser entendida como um processo de codificação e categorização de dados a fim de transformá-los em informação (DELLAGNELO; SILVA, 2005).

Contudo, não se adotou uma versão puramente quantitativa da análise de conteúdo, uma vez que o objetivo não era atribuir importância à recorrência de um item, mas sim a sua presença ou ausência dentro do planejamento estratégico. Para tanto, foi utilizado o método proposto por Bardin (2009), com algumas adaptações advindas de Krippendorff (2004).

\subsection{Definição Constitutiva e Operacional das Variáveis}

\subsubsection{Definição Constitutiva}

É possível encontrar uma série de definições na literatura para corrupção, sendo esse um tema ainda em discussão. A mais comum delas consiste em definir corrupção como o mau uso de um poder público para ganho particular (LAMBSDORFF, 2007). Também, a respeito de estratégia, são diversos os posicionamentos. Aqui se concebe estratégia como um padrão que guia uma corrente de decisões. Dentro dessa ótica, se adota o posicionamento cognitivista, sendo estratégia, portanto, um padrão formulado a partir de um processo mental, no qual é produzida uma visão do mundo que guia as linhas de ação (MINTZBERG et al., 2005).

Assim, pode-se conceber estratégias de combate à corrupção como um padrão de ações voltados para o combate do mau uso de recursos públicos para fins privados, tendo como base uma visão organizacional do que se entende por origem da corrupção. 


\subsubsection{Definição Operacional}

Uma das formas de operacionalizar um constructo para fins de análise de conteúdo consiste em ancorar a elaboração das categorias em estudos anteriores, de preferência que tenham suporte empírico (KRIPPENDORFF, 2004). Dessa forma, tendo por base a classificação proposta por Huberts (1988) de estratégias de combate à corrupção e buscando subsídio nos trabalhos de Kellner (2000) e Kayra (2008), a respeito das principais estratégias adotadas pelas Instituições Supremas de Auditoria, foram concebidas as seguintes classificações estratégicas e suas correspondentes definições:

Quadro 1 Dimensões estratégicas para combate à corrupção

\begin{tabular}{l|l|c|c|c}
\hline \multicolumn{1}{c|}{ Estratégia } & \multicolumn{1}{c|}{ Descrição } & (KAYRA, 2008) & (KELLNER, 2000) & (HUBERTS, 1998) \\
\hline Comunicação & $\begin{array}{l}\text { Promover a transparência e a comunica- } \\
\text { ção com a sociedade; }\end{array}$ & $\times$ & $\times$ & $\times$ \\
\hline Capacitação & Melhorar a gestão pública; & $\times$ & $\times$ & $\times$ \\
\hline Orientação & Orientar a sociedade; & $\times$ & $\times$ & $\times$ \\
\hline Repressão & Combater a fraude e a corrupção; & $\times$ & $\times$ \\
\hline Organizacional & Melhorar os processos internos; & $\times$ & $\times$ \\
\hline Política & $\begin{array}{l}\text { Melhorar a cooperação com legislativo e } \\
\text { outras instituições. }\end{array}$ & & $\times$ & $\times$ \\
\hline
\end{tabular}

Fonte: Elaborado pelos autores com base na classificação de Huberts (1988)

A partir dessa tipologia, que conjuga opinião dos especialistas com teoria, se buscou operacionalizar o constructo com base na verificação da presença e ausência das estratégias listadas nesses quadros nos planejamentos estratégicos dos Tribunais de Contas dos Estados.

\subsection{Hipótese de Pesquisa}

O que se propõe é a existência de uma diferenciação no tipo de estratégia em função do nível de renda per capita do Estado. Para justificar essa diferenciação são utilizadas as duas noções de natureza de corrupção apresentadas por Bukovansky (2006). Conforme a autora, a corrupção pode ser vista como um problema de agência, que se configura como algo inerente na relação entre agente e principal, ou como um problema estrutural em que há uma perda do senso de moralidade por parte da população.

Nesse sentido, em um contexto de alta renda, os órgãos de controle podem entender que a corrupção tem uma origem predominantemente oportunista, ao invés de moral, uma vez que, nesses ambientes, o indivíduo conta com uma melhor formação escolar e com um ambiente menos hostil. Nesse sentido, o indivíduo se sujeita à corrupção não necessariamente por um problema moral, mas por uma questão de incentivos. Assim, ações de natureza repressiva podem ser encaradas como uma melhor alternativa de ação em tal contexto.

Já para localidades de baixa renda há um ambiente com piores condições de vida para a população, havendo um sentimento coletivo de abandono do Estado, uma perda de uma identidade coletiva, por exemplo. É um ambiente mais hostil em que predomina uma lógica de ação individualista. Assim, pode-se conceber que o cidadão que se submete à corrupção o faz mais por um desvirtuamento moral do que por uma ação oportunista. Nesse cenário, a formulação estratégica pode passar por ações de natureza preventiva que estejam associadas a um desenvolvimento de uma ideia de integridade social.

\subsection{Categorização e Codificação}

Conforme Bardin (2009), a categorização é a etapa na qual os dados brutos são representados de forma simplificada. Ela deve obedecer aos princípios de exclusão mútua, homogeneidade, pertinência, objetividade/finalidade e produtividade. Também 
deve ser guiada pela hipótese de pesquisa que se deseja testar. Com isso em mente, foi realizada uma leitura flutuante dos objetivos estratégicos previstos nos planejamentos estratégicos dos TCEs a fim de se identificar os principais termos relacionados a cada categoria proposta. Dessa forma se construiu a seguinte associação para codificação:

Quadro 2 Dimensões estratégicas e termos pesquisados

\begin{tabular}{l|l}
\hline \multicolumn{1}{c|}{ Dimensão } & \multicolumn{1}{c}{ Termos pesquisados } \\
\hline Comunicação & $\begin{array}{l}\text { Transparência, comunicação, imagem, divulga- } \\
\text { ção, relacionamento; }\end{array}$ \\
\hline Capacitação & $\begin{array}{l}\text { Aperfeiçoamento, capacitação, melhoria, apri- } \\
\text { moramento, pedagógico; }\end{array}$ \\
\hline Educação & Orientação, controle social; \\
\hline Repressão & $\begin{array}{l}\text { Fiscalização, corrupção, fraude, desvio, irregula- } \\
\text { ridade; }\end{array}$ \\
\hline Organizacional & $\begin{array}{l}\text { Servidores/pessoas, procedimentos/processos, Tl, } \\
\text { estrutura, estrutura normativa, governança; }\end{array}$ \\
\hline Política & Parceria, cooperação, intercâmbio; \\
\hline
\end{tabular}

Fonte: Elaborado pelos autores

A codificação passa pela definição da unidade de registro, regra de enumeração e, em alguns casos, também pode incluir uma unidade de contexto se houver necessidade de evidenciar adequadamente a compreensão (DELLAGNELO; SILVA, 2005). No planejamento estratégico há uma enunciação direta dos objetivos estratégicos, não sendo necessária uma contextualização mais ampla para classificação.

Como unidade de registro são utilizados termos de pesquisa (Quadro 2). Contudo, há um ajuste de tema que consiste em não considerar os termos que não estejam relacionados com as ideias constituídas. A finalidade é verificar se as palavras pesquisadas representam a ideia contida na descrição da categoria de dimensões estratégicas. Nessa fase foram utilizados dois codificadores para evitar uma espécie de contaminação. Os codificadores realizaram a codificação dos termos nas categorias sem associá-los a qualquer teoria pré-definida.

Como regra de enumeração foi utilizado o critério de presença ou ausência dos termos. Assim, se buscou verificar se havia presença de, pelo menos, um dos termos associados a cada dimensão (cate- goria) estratégica, utilizando, portanto, o critério de presença e ausência.

Também foram criadas categorias nominais para as variáveis independentes a fim de se realizar as associações:

Quadro 3 Categorias das variáveis relacionadas à renda

\begin{tabular}{l|l|l}
\multirow{2}{*}{$\begin{array}{l}\text { Renda } \\
\text { per capita }\end{array}$} & Baixa renda & $\begin{array}{l}\text { 12 Estados com menores } \\
\text { Renda per capita }\end{array}$ \\
\cline { 2 - 3 } & Alta renda & $\begin{array}{l}\text { 12 Estados com maiores } \\
\text { Renda per capita }\end{array}$ \\
\hline \multirow{3}{*}{$\begin{array}{l}\text { Coeficiente } \\
\text { de Gini }\end{array}$} & Baixo Gini & $\begin{array}{l}12 \text { Estados com menores } \\
\text { Coeficiente de Gini }\end{array}$ \\
\cline { 2 - 3 } & Alto Gini & $\begin{array}{l}\text { 12 Estados com maiores } \\
\text { Coeficiente de Gini }\end{array}$ \\
\hline
\end{tabular}

Fonte: Elaborado pelos autores

\subsection{Análise}

Uma vez identificada a presença ou a ausência de cada estratégia, bem como sua natureza, foi realizada a análise gráfica de associação dessas categorias aos contextos de alta renda e baixa renda per capita.

Para realizar essa análise de forma exploratória foi utilizada a técnica de Análise de Homogeneidade (HOMALS), uma vez que ela permite obter um panorama visual das proximidades entre as categorias das variáveis em estudo (FÁVERO et al., 2009). Essa relação é evidenciada através de um mapa perceptual (ou mapa espacial) que mostra a posição relativa dos objetos (HAIR et al., 2005).

Através dela foi possível verificar a proximidade entre o tipo de estratégia e a dimensão renda per capita. Também foi possível identificar estratégias que, embora estejam relacionadas a uma categoria teórica, apresentam uma associação diversa daquela proposta pela teoria.

Por fim, de forma complementar, também se buscou a comprovação estatística da análise através de teste não paramétrico. $\mathrm{O}$ teste ideal proposto pela literatura para verificar se duas ou mais populações apresentam diferença com relação a uma determinada característica é o Qui-quadrado (RIFF et al., 2014). Contudo, o mesmo não deve ser aplicado quando as frequências esperadas são menores que o ideal $\left(\mathrm{E}_{\mathrm{ij}}<5\right)$ 
(SIEGEL; CASTELLAN JR., 2006), como ocorreu em algumas categorias. Nesse caso, como alternativa para inferência, deve ser empregado o teste exato de Fisher. Esse último é uma alternativa não paramétrica para comparar amostras independentes cujas frequências absolutas estejam agrupadas em tabelas de contingência (MARÔCO, 2011). Esse último teste foi aplicado, considerando-se uma probabilidade de erro do tipo I de $\mathrm{p}=0,05$ para todas as inferências.

\section{ANÁLISE DOS DADOS}

\subsection{Estatísticas Descritivas da Renda dos Estados}

O Brasil é um país que apresenta uma diversidade regional bem acentuada, principalmente no que se refere ao contexto de distribuição de renda e rendimento per capita. São encontrados indicadores de desigualdade típicos de países desenvolvidos (Gini de 0,43 em Santa Catarina) ao mesmo tempo em que também se verificam indicadores de elevada desigualdade $(0,63$ no Maranhão). Na renda a situação também é semelhante, sendo possível encontrar rendimento médio per capita que varia de R \$ 461,00 também no Maranhão, até R\$ 2.055,00 em Brasília (Tabela 1).

Tabela 1 Renda per capita e coeficiente de Gini dos Estados Brasileiros

\begin{tabular}{c|c|c|c|c|c}
\hline Est. & Gini & $\begin{array}{c}\text { Renda } \\
\mathbf{p} / \mathbf{c}\end{array}$ & Est. & Gini & $\begin{array}{c}\text { Renda } \\
\mathbf{p} / \mathbf{c}\end{array}$ \\
\hline AC & 0,487 & 670 & $\mathrm{PE}$ & 0,470 & 802 \\
\hline $\mathrm{AM}$ & 0,449 & 739 & $\mathrm{PI}$ & 0,568 & 659 \\
\hline $\mathrm{BA}$ & 0,530 & 697 & $\mathrm{PR}$ & 0,471 & 1.210 \\
\hline $\mathrm{CE}$ & 0,515 & 616 & $\mathrm{RJ}$ & 0,485 & 1.193 \\
\hline $\mathrm{DF}$ & 0,557 & 2.055 & $\mathrm{RN}$ & 0,504 & 695 \\
\hline $\mathrm{ES}$ & 0,475 & 1.052 & $\mathrm{RO}$ & 0,455 & 762 \\
\hline $\mathrm{GO}$ & 0,457 & 1.031 & $\mathrm{RR}$ & 0,481 & 871 \\
\hline $\mathrm{MA}$ & 0,633 & 461 & $\mathrm{RS}$ & 0,462 & 1.318 \\
\hline $\mathrm{MG}$ & 0,475 & 1.049 & $\mathrm{SC}$ & 0,430 & 1.245 \\
\hline $\mathrm{MT}$ & 0,488 & 1.032 & $\mathrm{SE}$ & 0,540 & 758 \\
\hline $\mathrm{PA}$ & 0,474 & 631 & $\mathrm{SP}$ & 0,468 & 1.432 \\
\hline $\mathrm{PB}$ & 0,515 & 682 & $\mathrm{TO}$ & 0,504 & 765 \\
\hline
\end{tabular}

Fonte: Renda per capita (R\$) (IBGE, 2014) e coeficiente de Gini (IBGE, 2012).
Contudo, quando considerados em conjunto o Brasil apresenta indicadores típicos de países em desenvolvimento (Gini médio de 0,496 e Renda p/c de $\mathrm{R} \$ 934,00)$. Assim, o país é propício para estudar organizações típicas de um país em desenvolvimento, mas que se encontram inseridas em diferentes realidades regionais de renda.

\subsection{Estatísticas Descritivas dos Planejamentos Estratégicos}

Os Tribunais de Contas Estaduais se encontram em diferentes estágios de evolução no que diz respeito a elaboração do planejamento estratégico. Enquanto alguns deles se encontram no primeiro ciclo de elaboração desses documentos, outros já se encontram na terceira versão do mesmo (DE ARAUJO, 2013).

Essa diversidade se reflete: a) no período abrangido pelo documento, que varia de 4 a 9 anos, tendo como média 5,3 anos; b) no número de páginas, que vai de 12 até 101 , tendo em média 47 e apresentando em alguns casos apenas uma versão do mapa estratégico no site; c) na realização de análise de pontos fortes/fracos e ameaças/oportunidades, que é feita por 14 deles de forma explícita no documento; d) pela adoção de indicadores de desempenho, adotada em 20 Tribunais; e) pela quantidade de indicadores, que varia de 12 a 49 , tendo como média 22,5 ; f) e pela quantidade de objetivos, que gira entre 3 a 23 , tendo como média 13,2 (Tabela 2).

Apesar dessa heterogeneidade foi possível destacar uma certa homogeneidade na metodologia aplicada, que em 18 casos explicitamente fez menção ao Balance Scorecard (BSC) e nos 6 demais, embora não deixasse explícita a utilização de tal metodologia, foi elaborado o mapa estratégico. Esses dados são apresentados a seguir: 
Tabela 2 Descrição dos Planejamentos estratégicos dos Tribunais de Contas Estaduais

\begin{tabular}{|c|c|c|c|c|c|c|c|}
\hline Est. & Período & Metodologia & Páginas & SWOT & $\begin{array}{l}\text { Indicador de } \\
\text { desempenho }\end{array}$ & $\begin{array}{c}\text { Quant. de } \\
\text { indicadores }\end{array}$ & $\begin{array}{l}\text { Quant. de Obje- } \\
\text { tivos }\end{array}$ \\
\hline$A C$ & $2011-2014$ & BSC & 60 & S & S & 18 & 12 \\
\hline AM & $2012-2016$ & BSC & 44 & S & $S$ & 20 & 12 \\
\hline BA & $2014-2017$ & $\mathrm{BSC}$ & 64 & S & S & 32 & 10 \\
\hline CE & $2010-2015$ & BSC & 63 & S & $S$ & 20 & 11 \\
\hline DF & $2011-2015$ & BSC & 35 & $N$ & S & 33 & 19 \\
\hline ES & $2010-2015$ & Não & 18 & $N$ & N & 0 & 16 \\
\hline $\mathrm{GO}$ & $2014-2020$ & BSC & Site & N & S & 42 & 23 \\
\hline MA & $2012-2016$ & BSC & 12 & N & S & 49 & 18 \\
\hline MG & $2015-2019$ & BSC & 28 & S & $\mathrm{N}$ & 0 & 14 \\
\hline MT & $2012-2017$ & BSC & 60 & N & $S$ & 19 & 13 \\
\hline PA & $2016-2021$ & BSC & 36 & S & S & 29 & 10 \\
\hline PB & $2011-2015$ & Não & 33 & S & S & 12 & 4 \\
\hline PE & $2013-2018$ & BSC & 54 & S & S & 26 & 11 \\
\hline $\mathrm{PI}$ & $2011-2015$ & Não & 66 & N & S & 17 & 3 \\
\hline$P R$ & $2008-2016$ & BSC & 101 & S & S & 28 & 17 \\
\hline RJ & $2012-2015$ & BSC & 20 & $S$ & $\mathrm{~N}$ & 0 & 10 \\
\hline $\mathrm{RN}$ & $2015-2021$ & BSC & 52 & $\mathrm{~N}$ & S & 21 & 13 \\
\hline $\mathrm{RO}$ & $2011-2015$ & BSC & 43 & $S$ & S & 12 & 12 \\
\hline $\mathrm{RR}$ & $2011-$ & Não & Site & $\mathrm{N}$ & $\mathrm{N}$ & 0 & 13 \\
\hline RS & $2015-2019$ & BSC & 93 & s & S & 39 & 17 \\
\hline SC & $2013-2016$ & Não & 20 & $N$ & S & 24 & 12 \\
\hline SE & $2011-2014$ & BSC & 40 & S & S & 17 & 14 \\
\hline SP & $2011-2015$ & Não & 27 & N & S & 49 & 18 \\
\hline TO & $2011-2014$ & $\mathrm{BSC}$ & 67 & $S$ & S & 34 & 15 \\
\hline
\end{tabular}

Fonte: Elaborado pelos autores

Como decorrência da utilização do BSC como metodologia, os Tribunais classificaram os objetivos estratégicos dentro de dimensões estratégicas (Tabela 3 ). No presente trabalho foram contabilizados nos 24 planejamentos analisados um total de 327 objetivos estratégicos que, em sua maioria (56\%), se encontram alocados dentro de uma dimensão de processos internos e pessoas, demonstrando uma orientação do Tribunal mais focada em uma atuação intraorganizacional. Esses dados refletem a constatação de Júnior e De Aquino (2011) de que há um predomínio de indicadores de quantidade de trabalho ao invés de resultados. 
Tabela 3 Dimensões estratégicas constantes nos planejamentos estratégicos

\begin{tabular}{l|c|c}
\hline \multicolumn{1}{c|}{ Dimensão Estratégica } & f & \% \\
\hline Processos internos e pessoas & 182 & $56 \%$ \\
\hline Resultado e Sociedade & 76 & $23 \%$ \\
\hline Recursos, orçamento e logística & 41 & $13 \%$ \\
\hline Aprendizado e Crescimento & 28 & $9 \%$ \\
\hline Total & $\mathbf{3 2 7}$ & $\mathbf{1 0 0}$ \\
\hline
\end{tabular}

Fonte: Elaborado pelos autores
Essa orientação para processos também se reflete na dimensão organizacional de combate à corrupção, na qual se verificou que a maioria dos Tribunais $(92 \%)$ tem um ou mais objetivos relacionados à melhoria de processos, otimização de seus recursos de tecnologia de informação, ampliação de suas estruturas físicas, busca por governança e modificações em estrutura de pessoal, tanto por meio de ampliação do número de servidores como pela implementação de gestão por competências (Tabela 4).

Tabela 4 Presença das dimensões estratégicas por Estado da Federação

\begin{tabular}{|c|c|c|c|c|c|c|c|}
\hline Est. & Comunic. & Capacitação & Educação & Repressão & $\begin{array}{c}\text { Organizacio- } \\
\text { nal }\end{array}$ & Política & Total \\
\hline$A C$ & $\times$ & & $x$ & $\times$ & $\times$ & & 4 \\
\hline AM & $x$ & & $x$ & & $\times$ & & 3 \\
\hline BA & & $\times$ & & $\times$ & $\times$ & $\times$ & 4 \\
\hline CE & $x$ & $x$ & & & $x$ & & 3 \\
\hline DF & $x$ & $x$ & $\times$ & $\times$ & $\times$ & $x$ & 6 \\
\hline ES & $\times$ & $x$ & & $\times$ & $\times$ & $x$ & 5 \\
\hline $\mathrm{GO}$ & $\times$ & $\times$ & $\times$ & $\times$ & $\times$ & $\times$ & 6 \\
\hline MA & $x$ & $x$ & $\times$ & $x$ & $x$ & & 5 \\
\hline MG & $x$ & $\times$ & $\times$ & $\times$ & $\times$ & & 5 \\
\hline MT & & $x$ & $x$ & $x$ & $x$ & & 4 \\
\hline PA & $\times$ & $\times$ & $\times$ & & $\times$ & $x$ & 5 \\
\hline PB & $x$ & & & & & & 1 \\
\hline PE & $x$ & $\times$ & $\times$ & & $\times$ & & 4 \\
\hline $\mathrm{PI}$ & $x$ & $x$ & $\times$ & $x$ & & & 4 \\
\hline PR & $x$ & $x$ & & $\times$ & $\times$ & $\times$ & 5 \\
\hline RJ & $x$ & $x$ & $x$ & & $x$ & $x$ & 5 \\
\hline $\mathrm{RN}$ & $\times$ & $x$ & $\times$ & $\times$ & $\times$ & & 5 \\
\hline RO & $x$ & $x$ & $x$ & $x$ & $x$ & & 5 \\
\hline RR & $\times$ & & $\times$ & & $\times$ & $\times$ & 4 \\
\hline RS & $\times$ & $\times$ & $\times$ & & $\times$ & $x$ & 5 \\
\hline SC & $x$ & $\times$ & & $\times$ & $\times$ & & 4 \\
\hline SE & $\times$ & & $\times$ & $\times$ & $\times$ & $\times$ & 5 \\
\hline SP & $x$ & $x$ & $\times$ & & $\times$ & & 4 \\
\hline TO & $x$ & & $\times$ & $\times$ & $\times$ & & 4 \\
\hline Total & 22 & 18 & 18 & 15 & 22 & 10 & Média \\
\hline$\%$ & $92 \%$ & $75 \%$ & $75 \%$ & $63 \%$ & $92 \%$ & $42 \%$ & 4,375 \\
\hline
\end{tabular}

Fonte: Elaborado pelos autores 
Interessante ressaltar que, apesar de prevalecer no Brasil um modelo de Corte de Contas, a dimensão de repressão (63\%) apresentou menor ocorrência que a dimensão de capacitação (75\%). O termo "corrupção", por exemplo, apareceu explicitamente apenas no planejamento de 5 Tribunais, os termos "desvio" e "irregularidade" em 7 e o termo "fiscalização" em 10.

Esse posicionamento parece refletir aquilo que os Tribunais têm entendido como sendo sua missão organizacional. Nesse sentido, em uma contagem de frequência das palavras que aparecem na missão apresentada nos planejamentos estratégicos, foi possível encontrar menção de palavras relacionadas à orientação em frequência superior à de fiscalização.

Tabela 5 Frequência das palavras constantes na missão dos Tribunais de Contas Estaduais

\begin{tabular}{l|c}
\multicolumn{1}{c|}{ Palavras } & Frequência \\
\hline Sociedade & 22 \\
\hline Gestão/Administração pública & 17 \\
\hline Controle externo & 15 \\
\hline Orienta* $^{*}$ & 13 \\
\hline Fiscaliz* $^{*}$ & 12 \\
\hline Controlar $^{\prime}$ & 6 \\
\hline Aperfeiçoando & 4 \\
\hline
\end{tabular}

Fonte: Elaborado pelos autores

* termos com diferentes terminações foram considerados.

Ex.: Orientação, orientativo, orientacional.

Essa orientação, contudo, apresenta certa variação entre os Tribunais, assim como as dimensões pelas quais eles trabalham para combater a corrupção. Na tabela 4 é possível identificar que apenas os Tribunais de Contas do Distrito Federal e de Goiás possuem ações classificadas nas 6 dimensões. Em média, os Tribunais trabalham com 4,3 dimensões, tendo alguns deles atuando apenas em uma. Isso posto, parece haver uma certa restrição na escolha entre quais linhas de ação atuar. Entender o que guia a escolha de uma ou outra dimensão estratégica é a que esse trabalho se propõe.

\subsection{Análise de Homogeneidade}

Para identificar como essas dimensões estratégicas se relacionam com as variáveis renda per capita e coeficiente de Gini, de forma exploratória, se procedeu à Análise de Homogeneidade (HOMALS). Essa é uma técnica que permite uma visualização de forma bidimensional de variáveis nominais, facilitando a visualização de associações entre as variáveis. É uma técnica equivalente à análise de componentes principais, no entanto, se diferencia por ser exclusiva para dados qualitativos (FÁVERO et al., 2009). A seguir são apresentados os eixos extraídos da aplicação da referida análise às categorias de dimensão, renda e coeficiente de Gini:

Tabela 6 Variância das dimensões extraídas

\begin{tabular}{l|c}
\multicolumn{1}{c|}{ Dimensão } & Valor Próprio \\
\hline 1 - Eixo Y & 0,286 \\
\hline 2 - EixoX & 0,195 \\
\hline
\end{tabular}

Fonte: Elaborado pelos autores

A tabela 6 mostra que as duas dimensões, quando consideradas conjuntamente, explicam $48,10 \%$ da variação dos dados (soma dos valores próprios de cada dimensão). A partir dessas duas dimensões é possível elaborar o mapa perceptual para verificar a posição relativa de cada variável. Quanto mais afastadas as variáveis de uma mesma categoria, maior sua discriminação. Ao passo que, quanto mais próximas as variáveis de categorias diferentes, mais associadas estão. As variáveis das categorias estratégicas, bem como as variáveis de renda per capita e do coeficiente de Gini são apresentadas a seguir: 
Figura 1 Mapa perceptual das dimensões estratégicas, renda per capita e coeficiente de GINI

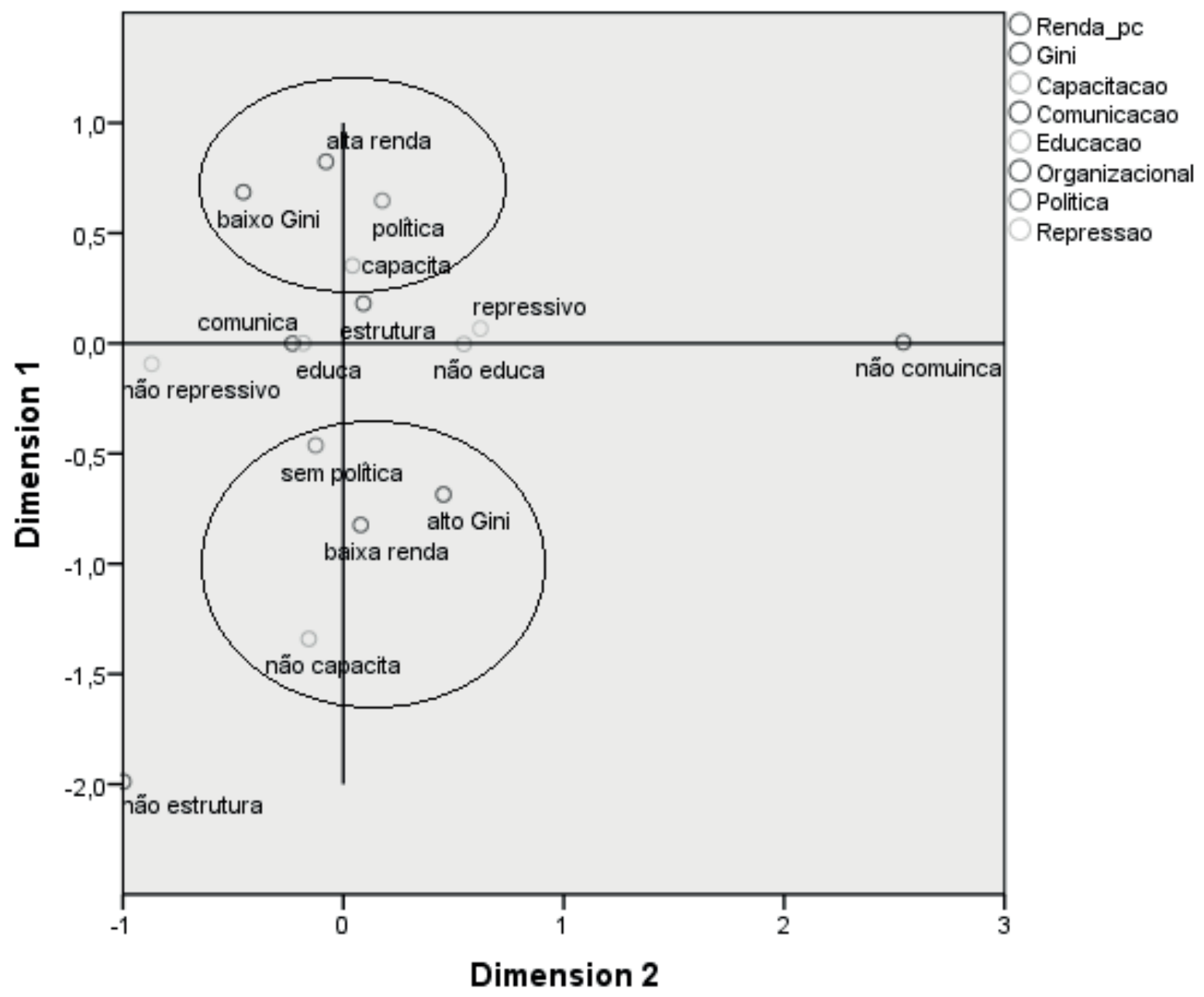

Fonte: Elaborado pelos autores

Através do mapa perceptual foi possível verificar que existe certa associação entre as dimensões estratégicas de atuação política e de capacitação com os indicadores demográficos de renda per capita e do coeficiente de Gini, principalmente devido à dimensão um. Verifica-se que, em contextos de elevada renda e baixa desigualdade, há a presença no plano estratégico de ações da capacitação e de atuação política junto a outras instituições, ao passo que em contextos de baixa renda per capita e maior desigualdade na distribuição de renda há a ausência de tais ações no planejamento estratégico dos Tribunais de Contas Estaduais.
Cabe ressaltar que essa associação tem caráter exploratório e descritivo, com o objetivo de levantar associações em um espaço multidimensional, não sendo passíveis de suportar inferências. Para tanto, em seguida foi realizada a confirmação dessas associações através de teste estatístico.

\subsection{Inferência Estatística}

Como mencionado, em função da ocorrência de casos de frequência relativa inferiores à 5 procedeu-se ao cálculo do teste exato de Fisher. Os dados são apresentados na tabela a seguir: 
Tabela 7 Teste exato de Fisher

\begin{tabular}{l|c|c}
\hline \multicolumn{1}{c|}{ Estratégia } & Coef. de Gini & Renda p/c \\
\hline Comunicação & 0,478 & 1,000 \\
\hline Capacitação & 0,317 & $0,037^{*}$ \\
\hline Educação & 1 & 1,000 \\
\hline Repressão & 0,68 & 1,000 \\
\hline Organizacional & 0,478 & 0,478 \\
\hline Política & 0,68 & 0,214 \\
\hline
\end{tabular}

Fonte: Elaborado pelos autores

Através da análise inferencial verificou-se que a proporção de ocorrência de estratégias de capacitação nos planejamentos estratégicos dos Tribunais de Contas é maior em Estados de alta renda $(\mathrm{p}=0,037)$. Com relação às demais dimensões estratégicas não foi identificada diferença estatística significativa, havendo, inclusive casos, em que a proporção é igual $(\mathrm{p}=1,000)$.

Todos os 5 Estados que não apresentaram políticas de capacitação da Administração Pública estão situados entre os 12 Estados de menor renda. Já todos os 12 Estados de maior renda preveem objetivo explícitos de capacitação (Figura 2).

Figura 2 Frequência de adoção de estratégias de capacitação conforme extrato de renda

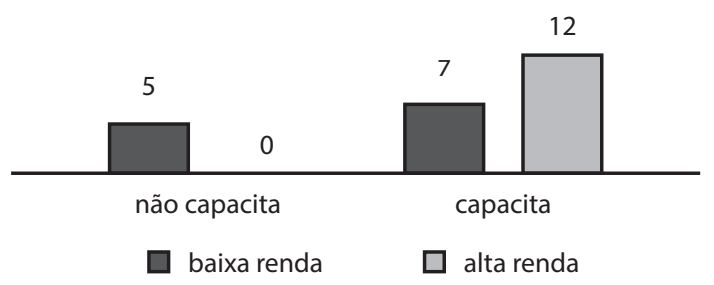

Fonte: Elaborado pelos autores

Essas estratégias de capacitação, contudo, quando aparecem, estão associadas à uma dimensão de processos internos, o que mostra uma diferença de atuação no combate à corrupção apenas na dimensão de atuação procedimental. Curiosamente, a dimensão capacitação não apresenta diferença significativa em contextos de desigualdade de renda $(\mathrm{p}=0,317)$.

\section{CONSIDERAÇÕES FINAIS}

Através dos dados levantados foi possível verificar que os Tribunais de Contas Brasilieiros apresentam em sua formulação estratégica outras dimensões de atuação além da típica função repressiva, evidenciando uma busca por alternativas de maior impacto social, seguindo uma tendência internacional (JOHNSEN et al., 2001; REICHBORN-KJENNERUD; JOHNSEN, 2015). Em que pese essa mudança de perspectiva, ainda prevalecem ações estratégicas tipicamente relacionadas à dimensão estratégica de processos internos ao invés da dimensão de resultado.

A hipótese inicial do trabalho propunha uma associação entre a presença de estratégias repressivas e contextos de alta renda, assim como entre presença de estratégias de capacitação e educação e contextos de baixa renda. Os dados, contraintuitivamente, mostraram que a presença de estratégias repressivas não está associada à renda e que a presença de estratégias de capacitação se relaciona com contextos de renda mais elevada.

Esses resultados sugerem que ações ligadas ao desenvolvimento de uma moralidade no setor público são mais presentes em contextos de elevada renda. Essa relação pode ser atribuída a uma percepção de que em ambientes de elevada renda a corrupção é um problema de natureza eminentemente moral, devendo os Tribunais agirem também de forma orientativa e preventiva.

Os resultados também apontam que de forma consciente ou não os Tribunais de Contas levam em consideração os aspectos ambientais em seu planejamento (KELLNER, 2000), bem como consideram a possibilidade de diferentes modelos de racionalidade dos jurisdicionados (HODGSON; JIANG, 2007) e, com isso, buscando a formulação de estratégias que levem a uma autuação mais efetiva (BLUME; VOIGT, 2011) .

Por fim, cabe destacar que o presente estudo teve um caráver exploratório e focado apenas em perspectiva objetivista dos dados apresentado nos Planajementos Estratégicos. Também o estudo se limita em uma perspectiva temporal restrita, em função do curto período em que o planejemanto estratégico formal vem sendo adotado por tais instituições. Nesse sentido, trabalhos futuros podem 
expandir as evidências aqui obtidas com trabalhos focados em aspectos subjetivos dos agentes envolvidos no processo de planejamento, bem como adotando uma perspectiva temporal mais ampla com o intuito de verificar o padrão ao longo do tempo.

\section{REFERENCIAS}

BARDIN, L. Análise de Conteúdo. In: (Ed.). Análise de Conteúdo: Edições 70/LDA, 2009.

BARZELAY, M. Central audit institutions and performance auditing: A comparative analysis of organizational strategies in the OECD. Governance, v. 10, n. 3, p. 235-260, 1997. ISSN 1468-0491.

BLUME, L.; VOIGT, S. Does organizational design of supreme audit institutions matter? A cross-country assessment. European Journal of Political Economy, v. 27, n. 2, p. 215-229, 2011. ISSN 0176-2680.

BRINGSELIUS, L. The dissemination of results from supreme audit institutions: independent partners with the media? Financial Accountability \& Management, v. 30, n. 1, p. 75-94, 2014. ISSN 1468-0408.

BUKOVANSKY, M. The hollowness of anti-corruption discourse. Review of International Political Economy, v. 13, n. 2, p. 181-209, 2006. ISSN 09692290. Disponível em: < http://www.tandfonline.com/ doi/abs/10.1080/09692290600625413 >. Acesso em: 02 mar. 2016.

DE ARAUJO, F. Adoção do planejamento estratégico pelos tribunais de contas brasileiros. Dissertação (Mestrado em Ciências Contábeis) Universidade Federal de Minas Gerais. Belo Horizonte: 2013.

DELLAGNELO, H. E. L.; SILVA, R. C. D. S. Análise de Conteúdo e sua aplicação em Administração. In: VIEIRA, M. M. F. e ZOUAIN, D. M. (Ed.). Pesquisa qualitativa em administração: teoria e prática: FGV, 2005.
FÁVERO, L. P. et al. Análise de dados. Modelagem multivariada para tomada de decisões. Rio de Janeiro: Campus, 2009.

GUPTA, S.; DAVOODI, H.; ALONSO-TERME, R. Does corruption affect income inequality and poverty? Economics of governance, v. 3, n. 1, p. 23-45, 2002. ISSN 1435-6104.

HAIR, J. F. et al. Análise multivariada de dados. 5. ed. Porto Alegre: Bookman, 2005. 593 p.

HODGSON, G. M.; JIANG, S. The economics of corruption and the corruption of economics: an institutionalist perspective. Journal of Economic Issues, p. 1043-1061, 2007. ISSN 0021-3624.

HUBERTS, L. W. J. C. What can be done against public corruption and fraud: Expert views on strategies to protect public integrity. Crime, Law and Social Change, v. 29, n. 2-3, p. 209-224, 1998. ISSN 09254994. Disponível em: < http://dx.doi.org/10.1023/ A\%3A1008348803253 >. Acesso em: 02 mar. 2016.

JOHNSEN, Å. et al. Performance auditing in local government: an exploratory study of perceived efficiency of municipal value for money auditing in Finland and Norway. European accounting review, v. 10, n. 3, p. 583-599, 2001. ISSN 0963-8180.

JONG-SUNG, Y.; KHAGRAM, S. A comparative study of inequality and corruption. American Sociological Review, v. 70, n. 1, p. 136-157, 2005. ISSN 0003-1224.

JÚNIOR, E. C.; DE AQUINO, A. C. B. Indicadores de desempenho em Entidades Fiscalizadoras Superiores: o caso brasileiro. Contabilidade Vista \& Revista, v. 22, n. 3, p. 15-40, 2011. ISSN 0103-734X.

KAYRAK, M. Evolving challenges for supreme audit institutions in struggling with corruption. Journal of Financial Crime, v. 15, n. 1, p. 60-70, 2008. ISSN 1359-0790. 
KELLNER, W. Supreme audit institutions and their role in fighting corruption. The Role of Bilateral Donors in Fighting Corruption, p. 167, 2000.

KRIPPENDORFF, K. Content analysis: an introduction to its methodology 2nd Edition. 2004.

LAMBSDORFF, J. G. How Corruption Affects Productivity. Kyklos, v. 56, n. 4, p. 457-474, 2003. ISSN 1467-6435. Disponível em: < http://dx.doi. org/10.1046/j.0023-5962.2003.00233.x > . Acesso em: 02 mar. 2016.

. The institutional economics of corruption and reform: theory, evidence and policy. Cambridge University Press, 2007. ISBN 1139464760.

MARÔCO, J. Análise estatística com oSPSS Statistics. ReportNumber, Lda, 2011. ISBN 9899676322.

MARTONE, C. L. Instituições, setor público e desenvolvimento: o caso do Brasil. Fundação Instituto de Pesquisas Econômicas (Fipe), 2007.

MAURO, P. Corruption and growth. The quarterly journal of economics, p. 681-712, 1995. ISSN 0033-5533.

MCCUSKER, R. Review of anti-corruption strategies. Australian Institute of Criminology, 2006. ISBN 1921185295.

MCDAVID, J. C.; HUSE, I. Legislator uses of public performance reports: Findings from a five-year study. American Journal of Evaluation, p. 7-25, 2011. ISSN 1098-2140.

MINTZBERG, H. et al. Strategy Safari: A Guided Tour Through The Wilds of Strategic Mangament. Simon and Schuster, 2005. ISBN 0743270576.

MOCAN, N. What determines corruption? International evidence from microdata. Economic Inquiry, v. 46, n. 4, p. 493-510, 2008. ISSN 1465-7295.
NEGIN, V.; RASHID, Z. A.; NIKOPOUR, H. The causal relationship between corruption and poverty: A panel data analysis. MPRA Paper, 2010.

PALDAM, M. Corruption and religion adding to the economic model. Kyklos, v. 54, n. 2-3, p. 383-413, 2001. ISSN 1467-6435.

POLLITT, C. Performance audit in Western Europe: trends and choices. Critical perspectives on accounting, v. 14, n. 1, p. 157-170, 2003. ISSN 1045-2354.

PORTA, R. L. et al. Trust in large organizations. National Bureau of Economic Research. 1996

REICHBORN-KJENNERUD, K.; JOHNSEN, A. Performance Audits and Supreme Audit Institutions' Impact on Public Administration The Case of the Office of the Auditor General in Norway. Administration \& Society, p. 1-25, 2015. ISSN 0095-3997.

RIFF, D.; LACY, S.; FICO, F. Analyzing media messages: Using quantitative content analysis in research. Routledge, 2014. ISBN 1135912017.

SIEGEL, S.; CASTELLAN JR., N. J. Estatística não-paramétrica para ciências do comportamento. 2. ed. Porto Alegre: Artmed, 2006. 448 p.

STAPENHURST, R.; KPUNDEH, S. J. Curbing corruption: Toward a model for building national integrity. World Bank Publications, 1999. ISBN 0821342576.

SVENSSON, J. Eight questions about corruption. The Journal of Economic Perspectives, v. 19, n. 3, p. 19-42, 2005. ISSN 0895-3309.

WU, Y.; ZHU, J. Corruption, anti-corruption, and inter-county income disparity in China. The Social Science Journal, v. 48, n. 3, p. 435-448, 2011. ISSN 0362-3319. 